\title{
Automation Monitoring With Sensors For Detecting Covid Using Backpropagation Algorithm
}

\author{
Pravin R. Kshirsagar ${ }^{*}$, Hariprasath Manoharan², Vineet Tirth ${ }^{3}$, Mohd Naved ${ }^{4}$, \\ Ahmad Tasnim Siddiqui ${ }^{5}$ and Arvind K. Sharma ${ }^{6}$ \\ ${ }^{1}$ Department of Electronics and Communication Engineering, AVNIET, Hyderabad, India. \\ [Email : pravinrk88@yahoo.com] \\ ${ }^{2}$ Department of Electronics and Communication Engineering, Audisankara College of Engineering and \\ Technology, Andhra Pradesh, India \\ [Email : hari13prasath@gmail.com] \\ ${ }^{3}$ Department of Mechanical Engineering, College of Engineering, King Khalid University, \\ Abha 61413, Saudi Arabia \\ [Email : vtirth@kku.edu.sa] \\ ${ }^{4}$ Department of Business Analytics, Jagannath University, Delhi-NCR \\ [Email : mohdnaved@gmail.com] \\ ${ }^{5}$ Department of Computer Applications, Sherwood College of Professional Management, Lucknow, India. \\ [Email : tasnim5@yahoo.com] \\ ${ }^{6}$ Department of Computer Science, University of Kota, India. \\ [Email : drarvindkumarsharma@gmail.com] \\ ${ }^{*}$ Corresponding author: Pravin R. Kshirsagar
}

Received January 12, 2021; revised May 12, 2021; accepted June 24, 2021; published July 31, 2021

\begin{abstract}
This article focuses on providing remedial solutions for COVID disease through the data collection process. Recently, In India, sudden human losses are happening due to the spread of infectious viruses. All people are not able to differentiate the number of affected people and their locations. Therefore, the proposed method integrates robotic technology for monitoring the health condition of different people. If any individual is affected by infectious disease, then data will be collected and within a short span of time, it will be reported to the control center. Once, the information is collected, then all individuals can access the same using an application platform. The application platform will be developed based on certain parametric values, where the location of each individual will be retained. For precise application development, the parametric values related to the identification process such as sub-interval points and intensity of detection should be established. Therefore, to check the effectiveness of the proposed robotic technology, an online monitoring system is employed where the output is realized using MATLAB. From simulated values, it is observed that the proposed method outperforms the existing method in terms of data quality with an observed percentage of 82 .
\end{abstract}

Keywords: Data quality, Intensity of detection, Robotic technology, Wireless sensors 


\section{Introduction}

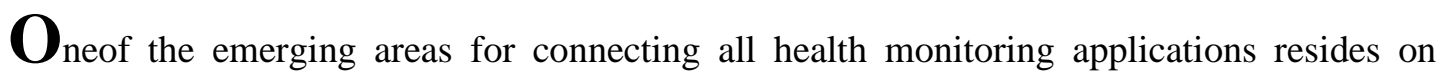
sensor-based technologies where all individuals can monitor themselves in every circumstance. This situation of self-monitoring ascends from the evolution of deadly diseases specifically for COVID. During such most horrible spread of diseases, humans cannot go in an outdoor environment and there is a need for every individual to monitor themselves. Therefore, the only way for each individual to monitor themselves is by using an intelligent device that produces results within a short period. It should be integrated with data-efficient processes. The use of wireless sensors in health applications overlays how senior citizensregularly monitor their health based onthe time-division process. Even the monitored conditions can be reported to consultants and in emergency circumstances, it can be directly reported to the control center for prior admittance in nearby hospitalities. This type of intelligent devices can save life of many individuals in emergency situations. Since the sensors perform many effectual assignments, it can be integrated with robotic technology to monitor outdoor conditions. Also, the environmental conditions of each sensor should be monitored to provide effective results as the diseases will be much higher during rainy period than seasonal periods. It should always be eminent that installed sensors should provide effective results with reduced energy consumption. In addition, severe breathing problems that occur due to COVID will only be monitored using oxygen saturation sensors and to control the spread of COVID, it is essential to wear concealment for better protection and this will also be continuously monitored using sensor-based technologies using an online monitoring system that is directly linked with control centers.

\section{Literature survey}

To analyze the effect of sensors on detecting COVID survey on existing literature has been examined. All examined literature has implemented different ways of monitoring COVID.However, the authors [1] have encompassed a drone technology for monitoring the affected patients in their corresponding countries. But if drone technology is introduced many drones have to employ with different address and the cost of implementation will become much higher. Wearable sensors have been introduced to identify the patients remotely [2] using simulated valuations. All metrics are measured using a commercial device where front line workers will be involved in this digital process for monitoring the parametric values. However, incorporating this digital technology will have a severe impact when data is shared among all networks (i.e.) there is no security concern allotted for the data aggregation process [2]. Also, a two-step strategy has been developed for identifying COVID-affected persons wherein, the first step calculation of immunity in each individual is carried out [3]. In the second step, a new type of biological receptor has been used for detecting the virus. Even though the accuracy of this two-step strategy is much higher, it cannot detect each individual through a speedy operation as optical fiber cables are used [3]. Additional, arguments have also been accomplished in analyzing the risk of COVID when all people are opened up and allowed to travel in each part of the world [4]. During this period, it is important to choose the kits for diagnosis to reduce the false detection of the virus. Therefore, the authors [4] suggest that the best method should be chosen for providing better results when society is ready to open up. But, a digital method of diagnosis has not been suggested and even the instructions focus on using a kit available at a local market. 
Similar to [3], a method of COVID detection in the nasopharynx has been introduced [5]. This type of method connects the transistor to biosensors where, graphing sheets which are present in the field-effect transistor will yield antibodies against COVID. In addition, the performance measure of this transistor is tested with a level of proteins in the human body. But the method of implementing this type of transistor will be much cost-effective and only biosensor will be installed in the human body without any relationship with transistors [5]. It is well known that only when people in society go out virus will supper and it is impossible to keep the same stage of lockdown during every period. Therefore, new smart helmets with sensors have been introduced [6] where, a thermal camera is fixed for taking the representation of every individual. In [6], the screening process will start by including a facial recognition technique. Therefore, if an individual has an abnormal temperature, their face will be captured and their entire details will be sent to healthcare administrations [6]. But no advanced system on a chip technology is integrated with the helmet and with a small camera inside the helmet, it is not possible to detect a human face with clear resolution [6].

It is identified that the spread of such viral disease happens due to travel. Therefore, immediate screening should be made with accurate calculations for the huge population [6]. For instant screening of patients, a new type of biosensor with more feasibility can be incorporated. By using biosensors, the huge population can be identified and at the same time, it averts all the affected patients to associate with other unaffected persons. Also, biosensors should be implemented after understanding comprehensive characteristics which include epidemiological and pandemic [8]. If the characteristics are not clear, then incorporating such a process to identify a transitional host will end up in the near future. Therefore, one best solution for such a process is to develop a label-free sensing technique by integrating smartphones, thus developing an immense library platform for identifying such diseases. But the use of nanotechnology equipment will provide more radiance, thus affecting each individual if preserved for a long period of time [7, 8]. To prevent the emission of exacting sensing equipment, it is sensible to use a fabric material made up of electrochemical units [9]. Thus, the probable capability to recognize even small organic parts will be much higher and paves the way for Nano viral identification. The incorporated electrochemical method will have more potential for identifying a considerable population within a short period of time using point of care technologies. Even it is admitted that electrochemical technologies will provide easy operation with a high probability of detection. However, the encumbrance of materials should be considered for proper installation. If more mass is provided, it is very difficult for the experts to carry it at a certain location where reasonable results cannot be obtained properly [9].

For the rapid increase in epidemic design, an information system is necessary for covering the entire geographic area [10]. Thus, new identification of diseases has paved the way for big data analysis, which gives rise to rapid visualization of images. Even the problems associated with data processing techniques can be solved using spatial analysis methods for entire data development. This type of geographical analysis is much easier than other methods [7-9]. But, data-driven approaches provide more advantage only in the central processing system and if it is incorporated for analyzing small data packets, then the cost of implementation will be a canned surplus. Consequently, an edge-driven approach using wireless networks can be developed for associating the data and all information about patients can be protected on incident nodes [11]. Similar to [9], a bio-safety material can also be incorporated for utilizing the model of material sciences [12] where an active arrangement between materials and biosafety can be provided at low cost. 
Even worst case arrangement should be considered for effective monitoring of different methodologies at minimum path resolution [13]. The method suggested in [13] is fairly similar to the proposed method but the major difference relies on converting the numerical functions obtained at high functionalities. Even the approach discussed in [13] is a non-linear function where the computation function is highly minimized. Moreover, it is much difficult to obtain a low-cost solution if the dimensions are greater because nanoparticles will be used and if a high dimension is combined, then it will be very difficult for the data monitoring process. The authors in [14] have combined the optimization and design process method where an in-depth analysis of the flow of network and data processing capabilities has been much more efficient than the procedures as mentioned above. But, the distribution of nodes is a challenging task when depth analysis has been considered [14]. For maintaining better ecology practices in health care services, it is necessary to use an advanced technology where data can be secured to a maximum extent [15]. This type of data security can be provided by blockchain technology where more feasible solutions can be obtained at less number of iterations. Also, if a better ecosystem is provided to patrons, then a healthier process will be maintained at it will be distributed to all individuals for proper identification of infections [15]. Additionally, diagnosis can be performed at a higher efficiency when neural networks are integrated [16] where even hidden layers can be tested at large data set. This type of multi-network model paves the way for attaining the best performance at minimum training time. But if a multi-model is used, then characteristics of the network should be defined sensibly and if any error is made during implementation, then entire network performance will be degraded [16-18].Additionally, recent literature [19-23] have also demonstrated that automatic detection of COVID is possible using different methods like neural network, X-ray images, radiography, deep learning and image encryption. Therefore, by considering the aforementioned concepts in cognizance a new method of COVID detection using block chain technology has been implemented in the proposed method.

\subsection{Research Gap and Motivation}

Some existing works have implemented a monitoring system for sensing individuals suffering from infectious diseases [1-16]. All existing methodologies have their benefits and problems addressed in deliberated methods are not adequate to implement in society as the enormous population is prevailing all over the world. So, for addressing the gap that occurs due to difficulty in the implementation process, the proposed method implements a robotic technology in addition to wireless sensors. The main motivation of the projected work is to sense information about people suffering from infectious disease and to provide safety precautions to the entire society where, automatons linkages will be placed in the general public. These robots will detect every individual and it will send reports to the control center. From the control center the monitored values will be tested and if any incongruities are established, then the individual will be separated within a short span of time.

To decipher the break-in existing literature, a back propagation algorithm that detects the hidden layer's value is incorporated. Also, to process the data in secured mode blockchain technology is introduced with the ethereum protocol. Thus the projected method is formulated as a multi-objective model where all parametric values can be monitored from the control center.

\subsection{Research Objectives}

The proposed method aims to detect each individual using a combined robotic sensor technology where the sensed information will be distributed to the control center from several 
locations, thus satisfying the following,

(i) High Strength of detection

(ii) Intensification of sub-interval points and

(iii) Minimization of the system cost.

\section{Problem formulation}

This section describes the mathematical model that is used for designing sensors to monitoring entire people around society. Since all sensors need to monitor in all directions (360 degrees), a bidirectional sensor is needed where the sensor function for sensing people can be designed using Equation (1).

Where,

$$
x_{i}(\text { sensor }, \text { object })=\left\{\begin{array}{lc}
1 & \text { if } x_{i} \leq a_{i} \\
0 & \text { Otherwise }
\end{array}\right.
$$

$a_{i}$ represents the area or circle of installed sensors

Equation (1) denotes that installed sensors will monitor the people and provide corresponding binary values ( 0 or 1$)$. If an individual is within a corresponding range, then a value of 1 will be indicated. If the same individual is out of range, it will also be detected by sensors and 0 will be specified. Also, there is a possibility that during detection, the corresponding individual will emanate and drive inside the area limits. At this time intensity of detection should be much higher which can be given as,

$$
I D_{i}=\max \sum_{i=1}^{N} x_{i}(\text { sensor }, \text { object })
$$

Where,

$I D_{i}$ denotes the intensity of detection and it should be exploited

Equation (2) indicates that the maximum number of sensors $(N)$ will be installed and its intensity should be much higher. This is very important in case of sensors because values will be detected more precisely only when the distance is much lesser. If the distance is larger, then, monitored values will be attenuated and will differ from original values. To avoid such circumstances, a bidirectional sensor is designed with a high intensity of detection. Even if the distance is larger then, two-directional tuples will be considered in the required direction as,

Where,

$$
x_{i}(\text { sensor }, \text { object })=\frac{(\cos (\varphi, \theta))^{\tau}}{\text { distance between sensor and object }}
$$

$\cos (\varphi, \theta)$ represents the two tuple values that lie within the angle of measurement $\tau$ denotes the corresponding attenuation which varies between 1-2 $\mathrm{dB}$

In the proposed method of detecting COVID whole area where all people live cannot be covered simultaneously. Therefore, it is important to partition all exposure paths by fixing the co-ordinates at starting an initial position which is considered as one end where a particular individual has started. Then, another end of the exposure path will be fixed on exact conflicting sideways. Thus, the term bi-directional sensor can be defined in the sensor field with a high dimensional equation as given in Equation (4). 
Where,

$$
P_{i}\left(b_{i}, \omega\right)=\int_{i=1}^{N} b_{i}(\vartheta) d l
$$

$b_{i}$ denotes the initial position of individual

$\omega$ represents the order of segregating all corresponding paths

$\vartheta$ is used to calculate the arbitrary distance between two points

Equation (4) indicates a non-linear equation which is solved only by segregating all considered paths into several sub-interval points as given in Equation (5).

Where,

$$
P_{i}\left(b_{i}, \omega\right)=\min \sum_{i=1}^{N} b_{i}(\vartheta) \Delta d_{i}
$$

$\Delta d_{i}$ denotes sub-interval points in considered paths

Equation (5) is a non-differentiable equation and it indicates that corresponding sub-interval points should be minimized for the correct data aggregation process. The process of spraying sanitizer by the robotic system depends on this process and it will work out perfectly when two paths, such as dispersion and destination, are clearly defined. If an individual is moving opposite to the installed sensor, then a minimum acquaintance value will be attained. It is also essential to consider the weight of the sensor at these two points of nodes where sensors are connected with each other. Thus, the weight of sensors in a permanent expanse can be given as,

Where,

$$
\sum_{i=1}^{N} w_{1}+w_{2}+. .+w_{i}=1
$$

$w_{1} \ldots w_{n}$ denotes the weight vectors

In the proposed method, different weight vectors will always be defined because each sensor will have different tasks. Thus in Equation (6), different weight sets are introduced. In addition, different sensing time for the data collection process should also be defined by sending and receiving data to corresponding robots. This varying time can be given as,

Where,

$$
t(i)=n t(i)+d t(i)+q t(i)
$$

$n t(i), d t(i)$ and $q t(i)$ represents the node, data transmission and queuing time of connected sensors.

Moreover, the production of sensors should be analyzed by calculating the cost of each sensor. This is important because all sensors have different weights with different tasks and it needs to sense a high volume of data. Therefore, the cost of installed sensors can be calculated as,

Where,

$$
\min c_{i}=\sum_{i=1}^{N} w_{i} v_{i}
$$

$v_{i}$ denotes variables that are used for the decision-making process

By combining Equation (1)-(8), the objective function of the proposed method can be defined in mathematical terms as, 
Where,

$$
O(i)=\min \sum_{i=1}^{N} c_{i}, P_{i}, \max \sum_{i=1}^{N} I D_{i}
$$

$C_{i}$ denotes the cost of sensor implementation

$P_{i}$ represents the initial position of bidirectional sensors that is obtained from Equation (4)

$I D_{i}$ represents the intensity that is acquired from Equation (2)

The objective function that is framed Equation (9) indicates that cost and sub-interval points should be minimized but at the same time, the intensity of detection should be maximized by robots. The values of $P_{i}\left(b_{i}, \omega\right)$ is acquired from Equation (4) and corresponding values for intensity that is detected by robots is obtained from Equation (2).

The objective function indicates that cost and sub-interval points should be minimized but at the same time, the intensity of detection should be maximized by robots.

\section{Implementation of Blockchain for COVID}

The main purpose of implementing blockchain for detecting COVID is that details of pretentious patients should be preserved and it should not spread to all people in different societies. This technology is integrated with the proposed method for providing data security so that panic among different individuals will be stopped. Since, it is possible to integrate a blockchain protocol with installed sensors, the ethereum protocol is implemented for smart COVID detection systems. Also, this protocol provides the best solution when it is integrated with machine learning algorithms. Fig. 1 shows the working model of ethereum for COVID detection.

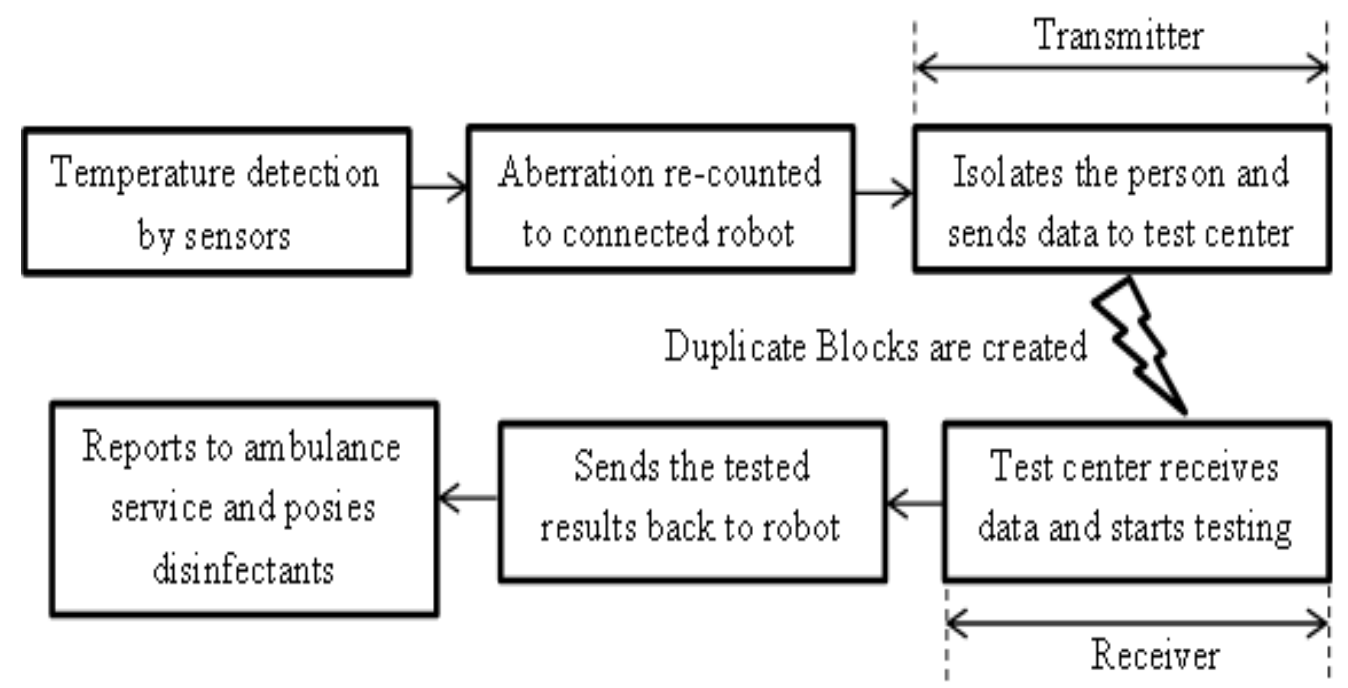

Fig. 1. Design of ethereum protocol for COVID.

The major reason for selecting the ethereum protocol is that it will adapt itself to different environments and be more flexible than other blockchain protocols. Moreover, ethereum is one of the most preferred protocols for back end data transfer process [15]. Even more complex model as per intellectual design can be prepared with this type of decentralized blockchain protocol. The working model of the ethereum protocol is designed so that a sensor 
will be positioned in suitable objects like a mask, helmet, or other objects that individuals are continually using. Therefore, the installed temperature sensor will monitor corresponding parameters and it reports to the robot which is treated as a receiver in corresponding sub-interval areas. If the robot detects any abnormalities, it will filter out that particular individual and directly reach the corresponding individual's location. In the proposed method, if an individual is identified, their home location will be identified. It will be reported to other nearest robots or if the affected individual stays within the same identified area, then the corresponding robot will reach the destination. Once the robot reaches the identified individual, then immediately disinfectant will be sprayed all around their body. A normal check will be made and the results will be sent to an authorized test center. If any abnormalities are detected, then immediately robot will automatically call nearby emergency services. In this case, more duplicate blocks will be created during the transmission and reception of data, so it becomes difficult for the hacker to steal the patient data.

To recognize this blockchain technology the difficulty in inspecting the process should be known. Thus, the difficulty can be defined using Equation (10) as,

Where,

$$
\sum_{i=0}^{N} B_{i}=\max \sum_{i=0}^{N} E_{i}
$$

$E_{i}$ denotes the maximum difficulty that is connected with blocks ranging from 0 to $N$

After calculating the number of difficulties, the probability rate should be calculated and for the proposed method, more number of blocks is created where, the numbers of attackers are found to be lesser. This can be denoted in mathematical form as,

Where,

$$
P_{i}(\beta)=\beta_{i}(N, 0.5)
$$

$\beta$ denotes that a partial data function for data segregation is used with a probability of 0.5 .

In the projected method, the autonomous robot will detect the original blockchain and move towards the target for disinfectant scattering. Thus, the identification of new targets by robotic technology can be found using Equation (12).

Where,

$$
t_{i}^{\text {new }}=t_{i}^{\text {old }} \times \frac{t(i)}{336 * B_{i} * E(t)}
$$

$t_{i}^{\text {new }}$ and $t_{i}^{\text {old }}$ denotes new and old targets identified by robots

$E(t)$ represents the protocol time which is equal to 600 seconds

The corresponding values of $t(i)$ and $B_{i}$ can be calculated from Equations (7) and (10) respectively. In this way, new targets are achieved within a short span of time with the creation of more duplicate blocks during the data transfer process.In Equation (12) protocol time defines the time period that is necessary for transmission and reception of certain information where, in the proposed method since the sensors are testing the health information of a particular person it is necessary that information should reach the receiver within small interval of time and is observed as 600 seconds. This timing has been generated after checking three different levels such as hardware, software and application levels and even the error rate in reception of packets is also lesser at time period of 600 seconds. 


\subsection{Optimization Algorithm}

The process of introducing robots for detecting COVID using sensors with ethereum protocol will perform efficiently only when the proper algorithm is chosen. The robot will not perform an automatic operation without the performance of neural network procedure. Therefore, for the effective functioning of the robot, a type of machine learning algorithm which is termed as back propagation algorithm is chosen. Similar to the ethereum protocol, the back propagation algorithm is also used for solving complex real-time problems. Since, in the proposed method, the input and output relationships are not known clearly. This algorithm is better to be implemented in robotic technology. In addition, the performance of this type of artificial algorithm is already examined in medical fields for detecting the response of patients during therapies. Moreover, the projected method wants to analyze digital images for COVID recognition. This requires high memory capacity storage and it will be provided by the back propagation algorithm. Further, one major advantage is that cost of processors will be much lesser where, dynamic information will be stored with only simple computations. Thus, this algorithm performs the exact sense of duty expected in sensing COVID and scattering sterilizers. Fig. 2 shows the relationship between input and output data in the back propagation algorithm.

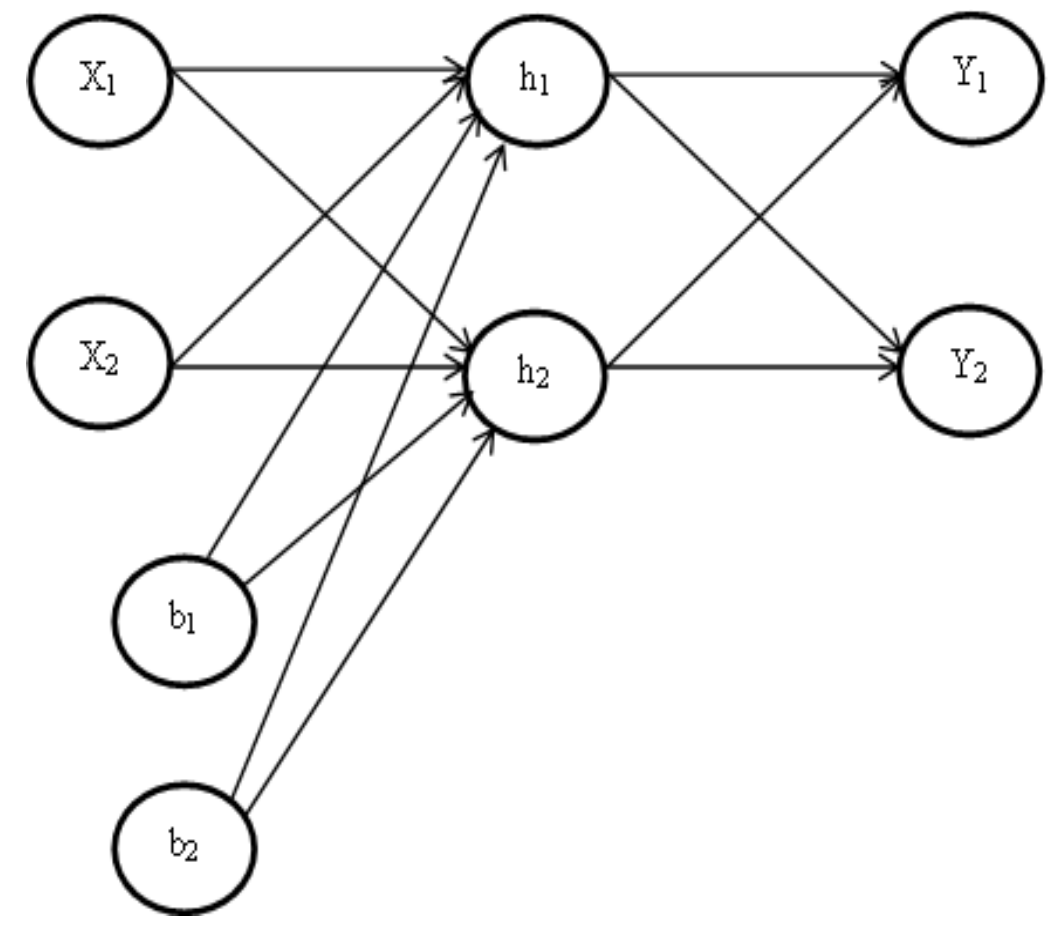

Fig. 2. Structure of back propagation algorithm.

From Fig. 2, it can be seen that two different paths are chosen and both are actuated using input vectors. Similar to expectancy, the signals will be broadcasted to all layers, thus introducing different neural networks. Fig. 3 illustrates the flow chart of integrating the back propagation algorithm for detecting COVID with blockchain technology. The task completed by different networks will be combined at the output stage with computational error as given in Equation (13). 
Where,

$$
\operatorname{fault}(i)=0.5 \sum_{i=1}^{N}\left(r_{i}-p_{i}\right)
$$

$r_{i}$ and $p_{i}$ denotes actual and probable output values with a probability of 0.5 .

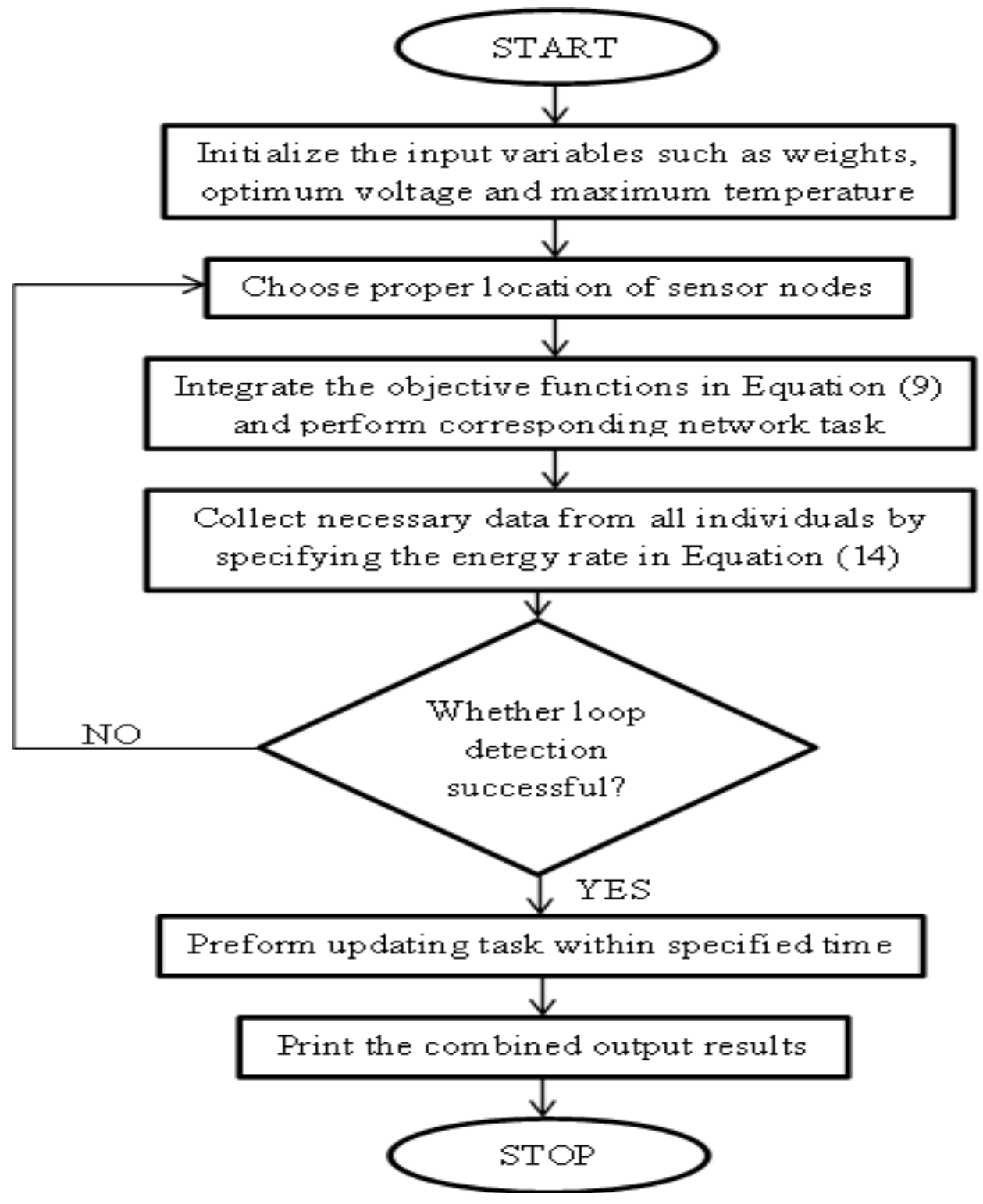

Fig. 3. Integration of machine learning for detecting COVID.

The purpose of calculating error is to analyze the effect of convergence. If the value of error is much smaller, then a faster convergence rate can be expected. In addition, for the back propagation algorithm, the learning rate should be much lower for achieving very small error values and to speed up the detection process. To achieve small error values, fluctuation in the entire network should be avoided. Therefore, the alpha energy rate should be defined which is given in Equation (14). 


$$
V_{i}=\frac{d E_{i}(t)}{d w_{i}}+\alpha E_{i}(t-1)
$$

Where,

$E_{i}(t)$ and $E_{i}(t-1)$ denotes the past and present energy values $\alpha$ represents the learning rate

In Equation (14) the value of learning rate will be defined after examining two different phases where, in the first phase larger values of alpha will be chosen between 0.3-0.9 and in the last phase i.e., at the end of simulation the values must be decreased at should range between 0.01-0.1. The aforementioned phases are considered because learning rate is a decreasing function of time and it should reach zero at the end of simulation.

\section{Results and Discussion}

For the proposed scheme data has been collected from an open research data set named as COVID-19 repository and for detecting the presence of COVID in human body three different sensor data will be aggregated and it will be monitored using a cloud based online monitoring system (Thing speak). Therefore, this cloud storage will be treated as base station for transmitting the health condition of each individual. From this cloud based storage only the observed parametric values are taken and simulated using MATLAB. This makes the proposed model more flexible because network cost will be reduced and even the specialists can monitor the health of individuals at their respective locations.

Table 1. Sensor arrangement

\begin{tabular}{|c|c|}
\hline Parameters & Specifications \\
\hline Electrochemical sensor \\
\hline Range of detection & $50-280 \mathrm{nM}$ \\
\hline Measuring distance & 40 Meters \\
\hline Temperature & 23.3 to 24 degrees (in centigrade) \\
\hline Size of sensor & $23 * 21 * 26 \mathrm{~mm}$ \\
\hline Operating voltage & 0 Volts (Unbiased) \\
\hline \multicolumn{2}{|c|}{ Wearable sensors } \\
\hline Range of detection & $445-455 \mathrm{nM}$ \\
\hline Measuring distance & 50 Meters \\
\hline Temperature & 20 to 42 degrees (in centigrade) \\
\hline Size of sensor & $3.05^{*} 2.1 * 1.1 \mathrm{~mm}$ \\
\hline Operating voltage & 2.6 to 3.5 volts \\
\hline \multicolumn{2}{|c|}{360 degrees } \\
\hline Range of detection & 30 Meters \\
\hline Measuring distance & $120^{*} 120^{*} 60$ mm \\
\hline Temperature & 10 Microvolts to 10 millivolts \\
\hline Size of sensor & \\
\hline Operating voltage & Bio sensors \\
\hline \multicolumn{2}{|c|}{} \\
\hline
\end{tabular}


To test the effectiveness of the projected robotic method using the back propagation, an online monitoring system is integrated. An online monitoring system results are simulated using MATLAB for effective analysis regarding variations of the following test scenarios.

Test scenario 1: Exploration of subinterval points

Test scenario 2: Testing the quality of data

Test scenario 3: Intensity of ailment exposure

Test scenario 4: Implementation cost

For the aforementioned four scenarios, all parametric data will be fed as input and the output will be calculated based on the different learning rates. All observed values will be designed using a three-dimensional plot using the etherum protocol.

\section{Test scenario 1}

In this scenario, one of the major challenges existing during sensor installation termed as sub-interval sensor allocation is analyzed. If the sensors are installed at the same frequency points in a cluster, then it is very easy to detect the necessary parameters within a short range of time. But in the proposed method, the results of COVID should pass the control center form different locations within a short span of time. For achieving such results, it is necessary to examine each sub-interval points and the sensors should be arranged placed by following different frequency spectrum. In other terms, the sensors should be designed with the capability of adjusting corresponding frequencies that are related to the corresponding areas. If this probability is achieved, then data that is collected from different locations will reach the control center at the proper time and consistent actions can be performed appropriately.

Fig. 4 shows the frequencies that are allocated to subinterval points according to different iteration values. Fig. 4 show that when numbers of iterations are increasing, the sub-interval points are changing randomly according to different areas. For example, if the number of iterations is equal to 5000, then allocated subinterval points will be 0.23 for the existing method. For the same area, the proposed method incorporates 0.27 for one cyclic period. The values for the proposed method is found to be higher because the area is clustered under remote locations. Therefore, more frequency is needed for collecting the data. Similarly, if the considered iteration value is 10000 and the considered area is not isolated from center point of the cluster, then the proposed method integrated 0.13 for one cyclic area whereas, for the same area the existing method [14] uses 0.17 which is much higher for the deliberated area. This proves that the wastage of sub-interval points is reduced when the proposed method is incorporated.

Also the results are compared with existing methods and they are observed in three dimensional approaches for achieving better indulgent. Since frequency is shared among sub interval points it enables more number of users to use same frequency bands which can be observed in Fig. 4. For same lowest frequency level which is observed to be between $50 \mathrm{~Hz}$ to $10 \mathrm{kHz}$ more number of users are present at same sub interval points which are varied in much lesser size. 


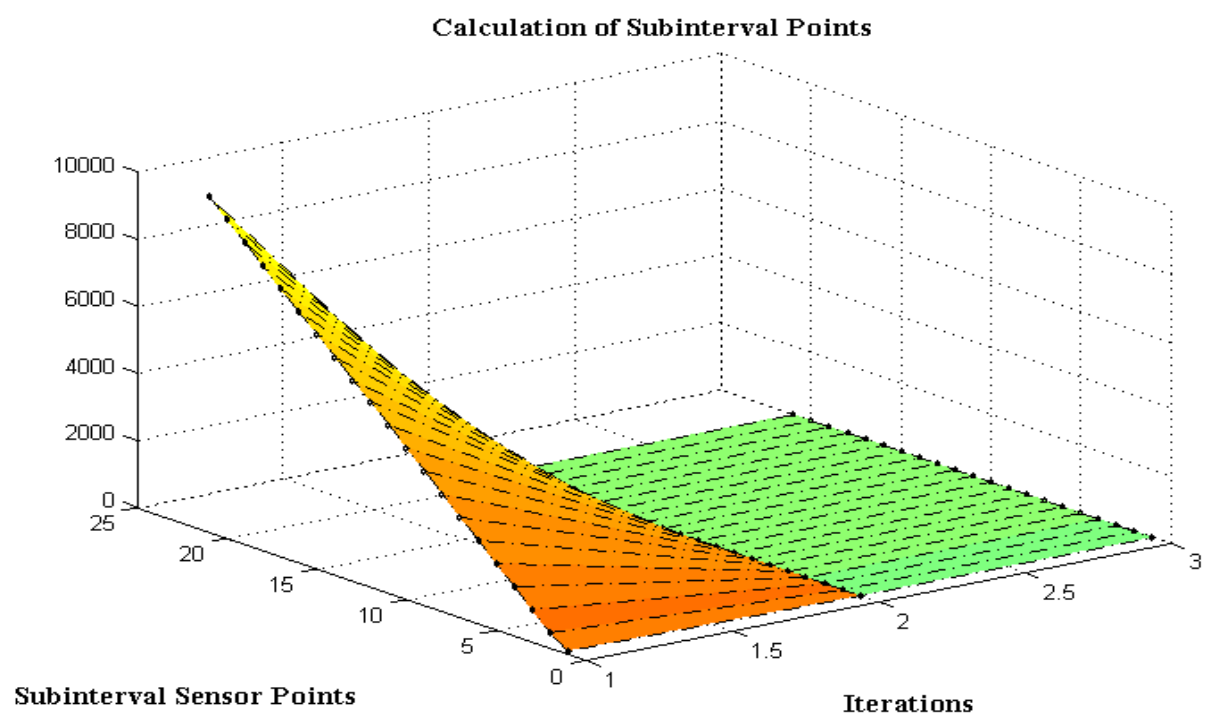

Fig. 4. Sharing of frequency at subinterval points.

Even with higher number of iterations between 6000 to 10000 the users continued to remain in same subinterval points which decidedly prove that the proposed method reduces the regularity wastage. In addition, natural form of frequencies remains persistent even when numbers of iterations are higher.

\section{Test scenario 2}

Once the sub-interval points are allocated, in the next step, it is essential to calculate the eminence of data that are collected. If wireless networks are integrated, then high-quality data should be ensured because as conversed in scenario 1, the sub-interval points are allocated only for collecting high-quality data. Therefore, if the quality of data is deprived, then accurate results cannot be obtained at any further stages. Fig. 5 shows the eminences of that are shared at each subinterval points with a number of sensor distribution.

From Fig. 5, it can be observed that the contour which is signifying the quality of data is much higher when numbers of sensors are increased. For example, if the number of installed sensors is 700 , then data eminence for the existing method will $63 \%$. For the proposed method, the percentage of data eminence is $78 \%$ which is much higher than the existing method [11]. Since the subinterval points are allocated correctly, this scenario demonstrates that the percentage of data eminence collected for sub-interval points are higher.Also, whenever IoT process is used in specific application then sensors should be in a position to collect high quality data and in this way data will be correctly detected and it is used for correcting errors. Additionally, Fig. 5 which is plotted using three dimensional pattern in order to observe microscopic changes that are happening when sensors are integrated. This process is necessary because for each millisecond the data will vary and if there are any reduction in quality of data then in the next sequence data rate of all sensors will become lesser. But physical layer which is present in the proposed method controls the bit synchronization using a common clock and transmission rate will be same for all packets that are sent by sensors which are indicated in right side of Fig. $\mathbf{5}$. 


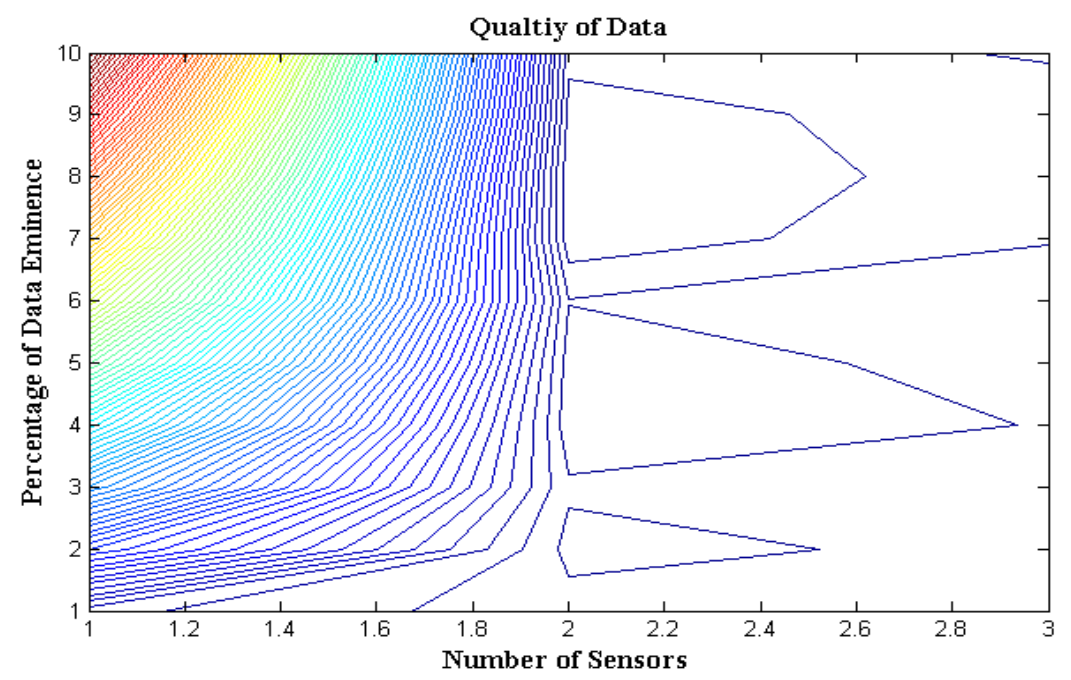

Fig. 5. Discerning data quality at sub interval points.

\section{Test scenario 3}

In this scenario, the intensity of sensor detection with respect to data quality is tested using the installed nodes. During COVID detection in several locations, more nodes will be connected where the intensity of each node should be higher. To be precise, a necessary amount of power should be supplied to sensors to sense different values of data. In many cases, the nodes that are connected to sensors may be higher but the amount of power supplied to connected nodes will be lesser.

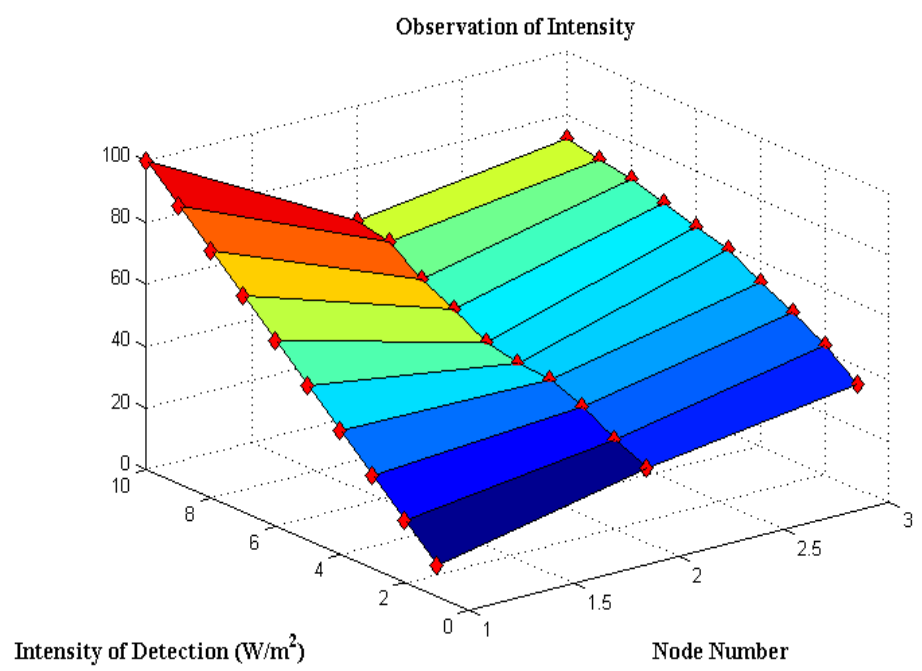

Fig. 6. Intensity of detection with sensor nodes

Fig. 6 shows the intensity that is detected for both existing [11] and proposed methods, which is expressed in watts per square meter. 
From Fig. 6, it can be perceived that even though the number of nodes varies between 10 and 100 , the proposed method supplies indispensable power without any wastage. For example, if the number of nodes is increased to 60 , then the supplied power should be maximized, which is equal to 62 watts per square meter. But for the same number of the sensor node, the existing method uses much less power as 42 watts per square meter. However, in practical applications, a higher number of nodes will not work with much lesser power for every meter allocated to different areas. Therefore, the proposed method proves to be much efficient in terms of intensity detection without any wastage. Once physical layer synchronized the data then data link layer will transmit the packets using error free communication channel. In this stage more number of bits that are sent by sensors will be converted into frames. The same process is integrated in the proposed method where after detecting the quality of data it is sent as frames to the next stage using intensity of progression. For identifying the rate of detection, node numbers will be used as reference and if more number of nodes are represented then a separate internet protocol address can be used.

\section{Test scenario 4}

Usually, the total cost in wireless networks will be calculated in accordance with a number of nodes but in the proposed method number of variables plays a major role. Therefore, all necessary node configurations are combined with the number of variables and the nodes are designed with respect to variables. Fig. 7 shows the total cost involved in the monitoring process, where the number of variables is varied between 5 and 20. The value of cost involved for all variables is expressed in INR. From Fig. 7, it can be seen even if the number of variables is high, the proposed method offers a low cost of installation when compared to the existing method [14].

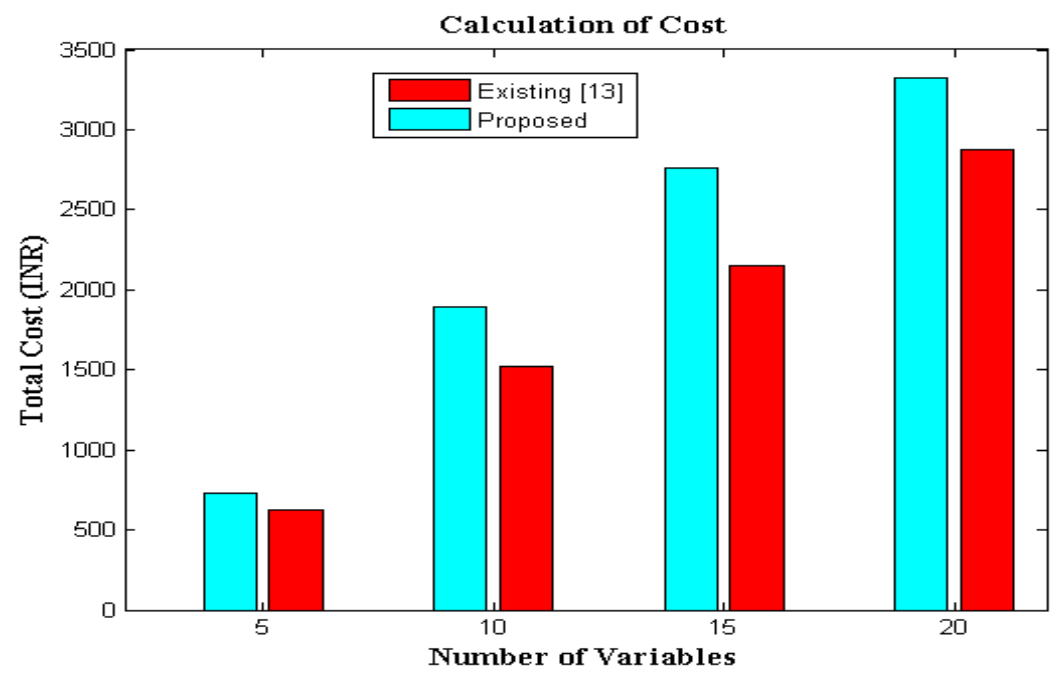

Fig. 7. Total cost in accordance with variables

For example, if the number of variables is 10 , the proposed method will be 1525 INR whereas with the same number of variables the existing method [13] offers 1893 INR which is much higher than the proposed method. Therefore, in all four scenarios, the proposed method proves to be more efficient in terms of intensity, cost and quality of data. 


\subsection{Performance criteria}

Since an intelligent device is installed for the purpose of detecting COVID it is necessary to check the convergence rate of sensors with respect to combined algorithm. If rate of convergence is achieved within less number of iteration then it is possible to define that the installed sensors is having the capability to provide test results at early stages. The same perception has been applied and it is tested using MATLAB where, the proposed method converges within less number of iterations when compared to existing method [10] as shown in Fig. 8.

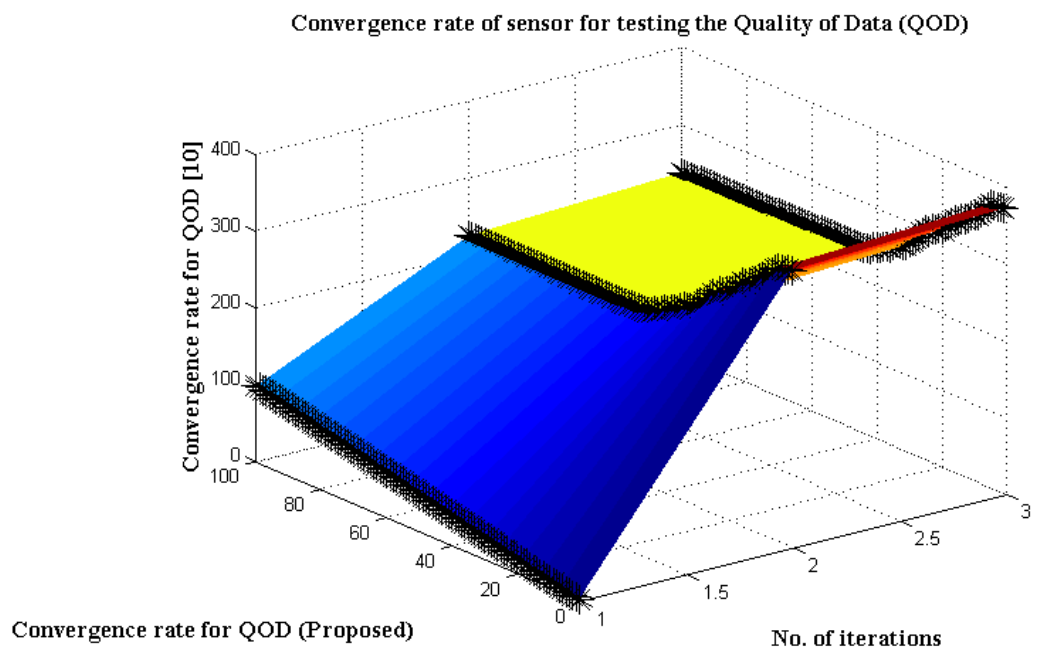

Fig. 8. Convergence optimization using QOD

From Fig. 8 it can be observed that the number of iterations is considered as 100 and convergence rate for testing the quality of sensors has been instigated. Once the process has been started the convergence of the proposed method decreases and once it reached 40th iteration a constant rate can be perceived. The same method has been applied to the existing method [10] but even though the convergence rate decreases, more number of iterations has been reserved. Therefore, the proposed method proves to be much efficient in terms of convergence rate when quality of data is being tested.

\section{Conclusions}

A new method of detecting pathological infections with sensors has been addressed in this article. Even though many researchers have found solutions for detection such as infectious diseases, a proper ailment with data processing techniques has not been implemented in many parts of the world. Therefore, it is necessary that data collection strategy about different people affected with toxic infections has to be analyzed and it should be reported to the control center for necessary actions. If the data collection strategy is reduced, it will be difficult to separate the affected individuals. No proper monitoring will be made, leading to the spreading of harmful diseases. The proposed method integrates a new methodology for placing intelligent monitoring devices in all areas where most of the individuals will be monitored in outdoor 
environments. To monitor each individual in an outdoor environment, the intensity of detection should be higher at each sub-interval point. If the sub-interval points are robust, then the data quality will be much higher which is achieved using the proposed method. For analyzing the effectiveness, four different scenarios are examined where, in each scenario, it can be observed that data quality is increased to the extent of $82 \%$ when compared with existing methods. If the proposed method is implemented in an outdoor environment, then information can be obtained within a short span of time, thereby reducing the risk of unaffected people.

\section{Mathematical Representations}

\begin{tabular}{|c|c|}
\hline Symbol & Representation \\
\hline$a_{i}$ & area or circle of installed sensors \\
\hline $\mathrm{ID}_{\mathrm{i}}$ & intensity of detection \\
\hline $\cos (\varphi, \theta)$ & two tuple values that lie within the angle of measurement \\
\hline$\tau$ & attenuation \\
\hline $\mathrm{b}_{\mathrm{i}}$ & initial position of individual \\
\hline$\omega$ & order of segregating all corresponding paths \\
\hline$\vartheta$ & arbitrary distance between two points \\
\hline$\Delta \mathrm{d}_{\mathrm{i}}$ & sub-interval points in considered paths \\
\hline $\mathrm{w}_{1} \ldots \mathrm{w}_{\mathrm{n}}$ & varght vectors \\
\hline $\mathrm{nt}(\mathrm{i}), \mathrm{dt}(\mathrm{i}), \mathrm{qt}(\mathrm{i})$ & node, data transmission and queuing time of connected sensors \\
\hline $\mathrm{v}_{\mathrm{i}}$ & variables that are used for the decision-making process \\
\hline $\mathrm{E}_{\mathrm{i}}$ & maximum difficulty that is connected with blocks ranging from 0 to $\mathrm{N}$ \\
\hline$\beta$ & partial data function for data segregation \\
\hline $\mathrm{t}_{\mathrm{i}}^{\text {new }}, \mathrm{t}_{\mathrm{i}}^{\text {old }}$ & new and old targets identified by robots \\
\hline $\left.\mathrm{E}_{\mathrm{i}} \mathrm{t}\right)$ & protocol time \\
\hline $\mathrm{r}_{\mathrm{i}}, \mathrm{p}_{\mathrm{i}}$ & actual and probable output values \\
\hline $\mathrm{E}_{\mathrm{i}}(\mathrm{t}), \mathrm{E}_{\mathrm{i}}(\mathrm{t}-1)$ & past and present energy values \\
\hline$\alpha$ & learning rate \\
\hline
\end{tabular}

\section{Acknowledgement}

The authors are thankful to the Institute of Research and Consulting Studies at King Khalid University (KKU), Abha-Asir, Kingdom of Saudi Arabia for supporting this research through grant number R.G.P.1/74/42.

\section{References}

[1] Y. Shen, D. Guo, F. Long, L.A. Mateos, H. Ding, Z. Xiu, R.B. Hellman, Adam King, S. Chen, C. Zhang and H. Tan, "Robots under COVID-19 Pandemic: A Comprehensive Survey," IEEE Access, vol. 9, pp. 1590-1615, 2020. Article (CrossRef Link)

[2] Seshadri DR, Davies E V., Harlow ER, et al., "Wearable Sensors for COVID-19: A Call to Action to Harness Our Digital Infrastructure for Remote Patient Monitoring and Virtual Assessments,” Front Digit Heal, 2, 1-11, 2020. Article (CrossRef Link) 
[3] Nag P, Sadani K, Mukherji S, “Optical Fiber Sensors for Rapid Screening of COVID-19,” Trans Indian Natl Acad Eng, 5, 233-236, 2020. Article (CrossRef Link)

[4] Pokhrel P, Hu C, Mao H, “Detecting the coronavirus (CoVID-19),” ACS Sensors, 5, 2283-2297, 2020. Article (CrossRef Link)h

[5] Seo G, Lee G, Kim MJ, et al., "Rapid Detection of COVID-19 Causative Virus (SARS-CoV-2) in Human Nasopharyngeal Swab Specimens Using Field-Effect Transistor-Based Biosensor,” ACS Nano, 14, 5135-5142, 2020. Article (CrossRef Link)

[6] Li X, Zhu L, Chu X, Fu H., "Edge Computing-Enabled Wireless Sensor Networks for Multiple Data Collection Tasks in Smart Agriculture,” J Sensors, 2020. Article (CrossRef Link)

[7] Mohammed MN, Hazairin NA, Syamsudin H, et al., "2019 novel coronavirus disease (Covid-19): Detection and diagnosis system using iot based smart glasses,” Int J Adv Sci Technol, 29, 954-960, 2020. Article (CrossRef Link)

[8] Kumar R, Nagpal S, Kaushik S, Mendiratta S., "COVID-19 diagnostic approaches: different roads to the same destination,” VirusDisease, 31, 97-105, 2020. Article (CrossRef Link)

[9] Chandra P., "Miniaturized label-free smartphone assisted electrochemical sensing approach for personalized COVID-19 diagnosis,” Sensors Int, 1, 100019, 2020. Article (CrossRef Link)

[10] Zhang W, Wang R, Luo F, et al, "Miniaturized electrochemical sensors and their point-of-care applications," Chinese Chem Lett, 31, 589-600, 2020. Article (CrossRef Link)

[11] Zhou C, Su F, Pei T, et al., “COVID-19: Challenges to GIS with Big Data,” Geogr Sustain, 1, 77-87, 2020. Article (CrossRef Link)

[12] Yu Y, Bu F, Zhou H, et al, "Biosafety materials: An emerging new research direction of materials science from the COVID-19 outbreak,” Mater Chem Front, 4, 1930-1953, 2020. Article (CrossRef Link)

[13] Binh NTM, Binh HTT, Van Linh N, Yu S, "Efficient meta-heuristic approaches in solving minimal exposure path problem for heterogeneous wireless multimedia sensor networks in internet of things,” Appl Intell, 50, 1889-1907, 2020. Article (CrossRef Link)

[14] Ibrahim A, Alfa A, "Optimization techniques for design problems in selected areas in WSNs: A tutorial,” Sensors (Switzerland), 17, 1-63, 2017. Article (CrossRef Link)

[15] Khatoon A, “A blockchain-based smart contract system for healthcare management,” Electron, 9, 2020. Article (CrossRef Link)

[16] Gupta A, Shreevastava M, “Medical Diagnosis using Back propagation Algorithm,” Int J Emerg Technol Adv Eng, 1, 55-58, 2011

[17] Al-Turjman F, Lemayian JP, "Intelligence, security, and vehicular sensor networks in internet of things (IoT)-enabled smart-cities: An overview,” Comput Electr Eng, 87, 106776, 2020. Article (CrossRef Link)

[18] Misganaw B, Vidyasagar M, "Exploiting Ordinal Class Structure in Multiclass Classification: Application to Ovarian Cancer,” IEEE Life Sci Lett, 1, 15-18, 2015. Article (CrossRef Link)

[19] T. Ozturk, M. Talo, E.A. Yildirim, U.B. Baloglu, O. Yildirim and U. Rajendra Acharya, "Automated detection of COVID-19 cases using deep neural networks with X-ray images," Computers in Biology and Medicine, vol. 121, pp. 103792, 2020. Article (CrossRef Link)

[20] M.Z. Islam, M.M. Islam and A. Asraf, “A combined deep CNN-LSTM network for the detection of novel coronavirus (COVID-19) using X-ray images,” Informatics in Medicine Unlocked, vol. 20, pp. 100412, 2020. Article (CrossRef Link).

[21] A. Kharrat, M. Halima Ben, M. Ben Ayed, "MRI brain tumor classification using Support Vector Machines and meta-heuristic method," in Proc. of International Conference on Intelligent Systems and Design Applications (ISDA), pp. 446-451, 2016. Article (CrossRef Link).

[22] M.K. Pandit, S.A. Banday, R. Naaz, M.A. Chishti, “Automatic detection of COVID-19 from chest radiographs using deep learning,” Radiography, vol. 27, pp. 483-489, 2021. Article (CrossRef Link).

[23] J. Khan, J.P. Li, B. Ahamad, S. Praveen, A.U. Haq, G. Ahmad Khan and A.K. Sangaiah, “SMSH: Secure surveillance mechanism on smart healthcare IoT system with probabilistic image encryption,” IEEE Access, vol. 8, pp. 15747-15767, 2020. Article (CrossRef Link). 


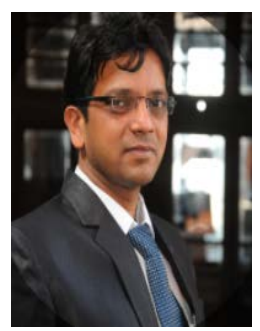

Dr. Pravin R. Kshirsagar presently serving as a Professor and Head-ECE in AVN Institute of Engineering \& Technology, Hyderabad, India. Previously, he served as a Vice Principal, Dean R\&D, Head-ETC in the in prominent Institute of India. He has vast experience of 18 years in teaching .He has served as reviewer in many international journals such as Inderscience, Spinger, Elsevier and IEEE Transaction. He has also delivered special talks in National \& International conferences and chaired various technical sessions in International conferences. He has published various research papers in reputed journals. He has published more than 40 Patents in National \& International levels. He has 6 books in his credential. He is profoundly engrossed in the area of Data Science, Machine Learning, Artificial Intelligence, and Computer Networks. Email: pravinrk88@yahoo.com

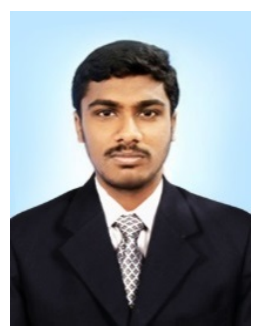

Dr. Hariprasath Manoharan is working as Assistant Professor in the Department of Electronics and Communication Engineering, and Associate Dean for Research and Development, Audisankara College of Engineering and Technology, Gudur, Andhra Pradesh, India. His areas of Research include Wireless Sensor Networks, Data Communications and Testing of Communication devices. He has published 13 research articles which includes SCI, SCIE, ESCI, SCOPUS indexed articles and has presented articles in 6 International Conferences. He has completed 4.3 years of Research experience and Teaching Experience. He has guided both B. Tech and M. Tech students for doing projects in the areas of Wireless Sensor Networks. He has also published a book entitled 'Computer Aided State Estimation for Electric Power Networks' which provides a complete guide to all Research Scholars in the field of Electronics and Communication Engineering. Email: hari13prasath@gmail.com

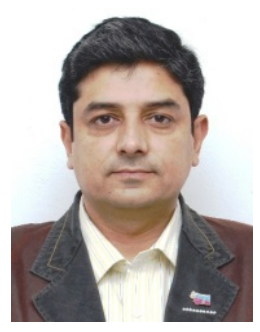

Dr. Vineet Tirth, Ph.D., is working as Associate Professor inMechanical Engineering Department, College of Engineering, King Khalid University, Abha, Kingdom of Saudi Arabia since 2015. Formerly he has worked as Professor in Mechanical Engineering and Director at Moradabad Institute of Technology, India. He has over 50 research publications, 5 patents and more than 25 presentations and invited talks in conferences and seminars. He has been the member of the board of studies and advisory committees of many institutions. He has completed 15 sponsored research projects to date and presently he is working on five projects. He is a member of Institution of Engineers, Indian Society for Technical education and many more. He is a reviewer of many international journals of repute viz. ASME Transactions, Metallurgical Transactions A, Measurements, Jr. of engineering Research, ASM Jr of Materials Engineering and Performance etc. He has organized many national and international conferences and won many awards in his academic and professional life. Email: vtirth@kku.edu.sa

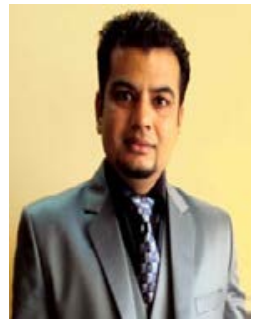

Dr. Mohd Naved is a machine learning consultant and academician, currently teaching as Assistant Professor and HoD (Analytics \& IB) in Jagannath University in collaboration with Xcelerator Ninja (India) for various UG \& PG programs in Analytics and Machine Learning. A former data scientist and an alumnus of Delhi University. He holds a PhD from Noida International University. He is actively engaged in academic research on various topics in artificial intelligences and 21st century technologies. His interviews have been published in various national and international magazines. 


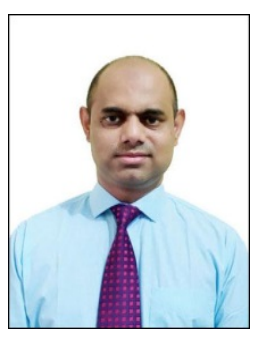

Dr. Ahmad Tasnim Siddiqui is an Assistant Professor in Department of Computer Applications at Sherwood College of Professional Management, Lucknow, India. He holds $\mathrm{PhD}$ (Computer Science) from OPJS University, India, M.Phil (Computer Science) and Masters of Computer Applications from Madurai Kamaraj University, India. He has 12 years of academic work experience and almost 5 years of software development experience. He has attended number of national and international conferences, seminars and workshops. He has published number of papers in reputed journals and published a book chapter in Emeralds Insight. His research interests include cloud computing, web mining, e-learning, IoT and e-commerce. He is also reviewer of some reputed journals like International Journal of Advanced Computer Science and Applications (IJACSA). Email: tasnim5@yahoo.com

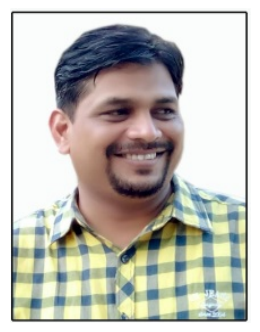

Dr. A. K. Sharma holds Ph.D and M.Tech degree in Computer Science from Rajiv Gandhi Proudyogiki Vishwavidyalaya (RGPV) Bhopal, Madhya Pradesh, India. He has more than 18 years of work experience in academic fields. He has supervised $5 \mathrm{Ph}$.D scholars and currently supervising 4 Ph.D scholars. He published more than 100 International Conferences and Journal papers. He has authored and co-authored almost 5 books on Computer Science so far. He has participated as Convener, Resource Person, Keynote Plenary Speaker, and Session Chair in many reputed National and International Conferences in India and abroad. He is also a Reviewer for more than 10 Journals such as Elsevier Journal of King Saud University, Computer \& Information Sciences, Springer Journal of Ambient Intelligence and Humanized Computing, IEEE Access, IGI Global, Asian Journal of Research in Computer Science, International Journal of Machine Learning and Computing, IJACSA, IJCST and so on. His research interests are Web Mining, Web Intelligence, Big Data Analytics, Internet of Things, and Machine Learning. Email: drarvindkumarsharma@gmail.com 